\title{
Evaluation of Omeprazole in the Treatment of Laryngopharyngeal Reflux Disease: A Single Center, Prospective and Randomized Study
}

\author{
${ }^{1}$ Kartik Doshi, ${ }^{2}$ Ashish Varghese, ${ }^{3}$ Dinesh K Badyal
}

\begin{abstract}
Aims: To assess efficacy and safety of omeprazole in laryngitis due to laryngopharyngeal reflux disease (LPRD).

Materials and methods: It was a prospective, randomized, open label study conducted in 90 patients with laryngitis due to LPRD. Group A $(n=45)$ received omeprazole and advice about life style modification, group $B(n=45)$ received only advice about life style modification. Reflux symptom index (RSI), reflux finding score (RFS), quality of life (QoL) and voice handicap index (VHI) were evaluated at 0,4 and 8 weeks.
\end{abstract}

Results: Percentage reduction in RSI and RFS was significantly higher in group A after 8 weeks (37 vs 29\%, 18 vs 13\% respectively). Excess throat mucus, clearing of throat and erythema of posterior larynx improved considerably in group $A$ compared to group B.

Conclusion: Omeprazole could be a significant addition to life style modification in LPRD patients particularly for signs and symptoms, such as excess throat mucus, clearing of throat and erythema of posterior larynx.

Keywords: Laryngitis, Laryngopharyngeal reflux, Omeprazole, Proton-pump inhibitors.

How to cite this article: Doshi K, Varghese A, Badyal DK. Evaluation of Omeprazole in the Treatment of Laryngopharyngeal Reflux Disease: A Single Center, Prospective and Randomized Study. Int J Otorhinolaryngol Clin 2015;7(2):45-50.

Source of support: Nil

Conflict of interest: None

\section{INTRODUCTION}

Laryngopharyngeal reflux disease (LPRD) occurs due to the backflow of gastric contents in to the larynx and hypopharynx. ${ }^{1}$ Prevalence of LPRD in otolaryngology clinics is around 4 to $10 \%$ while in primary care practice

\footnotetext{
${ }^{1}$ Senior Resident, ${ }^{2,3}$ Professor

${ }^{1,3}$ Department of Pharmacology, Christian Medical College Ludhiana, Punjab, India

${ }^{2}$ Department of Otolaryngology, Christian Medical College Ludhiana, Punjab, India

Corresponding Author: Kartik Doshi, Senior Resident Department of Pharmacology, Christian Medical College Ludhiana, Punjab, India, Phone: 7506169402, e-mail: kartik. hdoshi@gmail.com
}

is around $1 \% .^{2}$ Laryngopharyngeal reflux disease is diagnosed by clinical symptoms and signs. These symptoms are evaluated by reflux symptom index (RSI), while signs of LPRD are evaluated by Reflux finding score (RFS). ${ }^{3,4}$ Management of LPRD is divided into nonpharmacological, pharmacological and surgical modalities depending on the severity and response of the patients. Nonpharmacological measures include: life style changes, hydration therapy and speech therapy. Life style changes comprise of diet changes, weight reduction and sleeping pattern. ${ }^{5}$ Hydration therapy prevents dryness of vocal cords while speech therapy is given in LPRD patients for the correct use of voice. Pharmacological management includes drugs, such as proton pump inhibitors (PPIs), $\mathrm{H}_{2}$-receptor blockers, prokinetic agents, mucosal cytoprotectants, antihistamines and alginates. Proton pump inhibitors and $\mathrm{H}_{2}$-receptor blockers decrease gastric acid and thereby decrease the damage to laryngeal mucosa. Proton pump inhibitors like omeprazole, esomeprazole, pantoprazole, rabeprazole are given twice daily for at least 2 months. All the PPIs have comparable efficacy profile. ${ }^{6}$ Despite the widespread clinical practise of using PPIs in LPRD, the status of PPIs in LPRD is debatable. There are no clear cut guidelines for the management of LPRD. Several randomized controlled studies have shown that efficacy of PPIs are equivalent to placebo group. ${ }^{7-9}$ However, few studies have also shown benefit of giving high dose PPIs over placebo. ${ }^{10,11}$ One of the reasons for failure of PPIs is that the underlying causes of LPRD are multifactorial. ${ }^{12}$ It is unlikely that acid reflux is the sole mechanism, although an important one. Pepsin is the most important factor to be associated with LPRD but PPIs have no role in inhibiting pepsin. ${ }^{13}$

In view of the debatable data pertaining to the role of PPIs in LPRD, this study was planned to evaluate the efficacy of omeprazole in laryngitis due to LPRD.

\section{MATERIALS AND METHODS}

\section{Study Design and Protocol}

It was a prospective, randomized, comparative, and open labeled study. Patients were enrolled in the study after they signed a written informed consent. The study was approved by Institute Ethics Committee. The 
study was registered in Clinical Trial Registry of India (CTRI/2015/02/005501). All procedures contributing to this work comply with the ethical standards of the Indian Council of Medical Research (ICMR) on human experimentation and with the Helsinki declaration of 1975 , as revised in 2008. ${ }^{14,15}$

Patients were randomly divided into two groups: groups $\mathrm{A}$ and $\mathrm{B}$, by computer generated random numbers. Group A $(n=45)$ patients received omeprazole $(40 \mathrm{mg})$ twice daily for 8 weeks. In addition to this, they were advised about life style modification. Group B $(n=45)$ patients were advised about life style modification only without omeprazole. Patients from both groups received symptomatic treatment in the form of antihistaminics, analgesics, etc. Life style modification advice, such as weight loss, smoking cessation, avoidance of alcohol were given to all patients and also reinforced in subsequent visits. Patients were asked to restrict consumption of chocolates, fatty foods, carbonated beverages, spicy foods and late night meals. Patients were evaluated after 4 weeks of treatment and those who were not able to follow and/or benefitted from life style modification were given treatment in form of other PPIs.

\section{Participants}

Patients of both sex with age between 18 and 70 years having symptoms of LPRD since 1 month and RSI score $>13$ and RFS score $>7$ were included in the study. While patients taking concomitant medications including ketoconazole, theophylline, antacids and digoxin were excluded from the study. Patients of laryngitis due to other causes like bacterial or viral infections were also excluded from the study. Life-threatening complications of laryngeal, pharyngeal or esophageal cancer, subglottic or tracheal stenosis, laryngeal papilloma, airway compromise which can complicate the LPRD diagnosis were excluded from the study. Further exclusion criteria included patients with gastroparesis, previous endoscopic or surgical antireflux procedure or any gastric surgery. Finally, pregnant and lactating mothers were not enrolled in the study.

\section{Patient Flow and Follow-up}

Patients were assessed for RSI, RFS, VHI and QoL at baseline, 4 weeks and at end of study at 8 weeks. Reflux symptom index is a recently developed 9-item, 0 to 45 score, standardized, disease-specific outcome instrument for LPRD. ${ }^{3}$ Normative data suggest that an RSI score of up to 10 is normal, while a score greater than 13 suggests LPRD. Reflux finding score is used to quantify and standardize endoscopic findings of LPR. ${ }^{4}$ The RFS ranges from a minimum score of 0 (no inflammation) to a maximum score of 26; a score greater than 7 suggests the presence of LPRD. Voice handicap index-10 is a 10 item, shortened version of the original 30-item vocal handicap index. ${ }^{16}$ The VHI-10 represents a distilling of 3 sub-scales into one shortened scale. For each question the patient is required to rate each answer using a 5-point scale. The total score is used to indicate the severity of the voice disorders for the patient. Voice specific QoL index is total 30 number score. A score more than 5 indicates significant vocal disability. ${ }^{17}$ All patients underwent a thorough clinical examination including history, vital signs and systemic examination. All relevant investigations were done in clinically suspicious patients to rule out any other causes of laryngitis and laryngeal cancer. Patients were monitored for any adverse drug reactions (ADRs) according to the ADR check list and by voluntary reporting.

\section{STATISTICAL ANALYSIS}

Difference of score between two groups were analysed by Mann-Whitney U test. Within group analysis was done using Wilcoxon signed-rank test. Continuous parameters were compared using student-t test $\mathrm{p} \leq 0.05$ was considered as statistically significant. We analyzed the data as per intention to treat (ITT) analysis to determine the significance of adding PPIs in LPRD patients. We analyzed the data as per last observation carry (LOC) forward and ITT analysis.

\section{RESULTS}

A total of 90 patients diagnosed with laryngitis due to LPRD according to RFS and RSI criteria were included in the study. The demographic profile and baseline clinical characteristics were comparable in both groups (Table 1). The consort diagram of study depicts the number of patients screened and enrolled in the study (Flow Chart 1). Acid reflux symptoms were higher in group A

Table 1: Baseline demographic and clinical characteristics of the patients $(n=90)$

\begin{tabular}{lll}
\hline Characteristics & $\begin{array}{l}\text { Group } A \\
\text { (omeprazole) }\end{array}$ & $\begin{array}{l}\text { Group B (life style } \\
\text { modification) }\end{array}$ \\
\hline Total no. of patients & 45 & 45 \\
Age in (years) & $43.60 \pm 2.19$ & $38.44 \pm 1.89$ \\
Sex (M:F) & $24: 21$ & $24: 21$ \\
Mild acid reflux & 21 & 12
\end{tabular}

( $<3$ times/week)

Presenting laryngeal symptom

Hoarseness of voice

Excess throat mucus

Clearing of throat

FB sensation in throat

Pain in throat

$\begin{array}{ll}16(36 \%) & 18(40 \%) \\ 5(12 \%) & 7(16 \%) \\ 3(7 \%) & 6(14 \%) \\ 6(14 \%) & 4(9 \%) \\ 7(16 \%) & 5(12 \%)\end{array}$


Flow Chart 1: Consort diagram showing flow of participants in the study

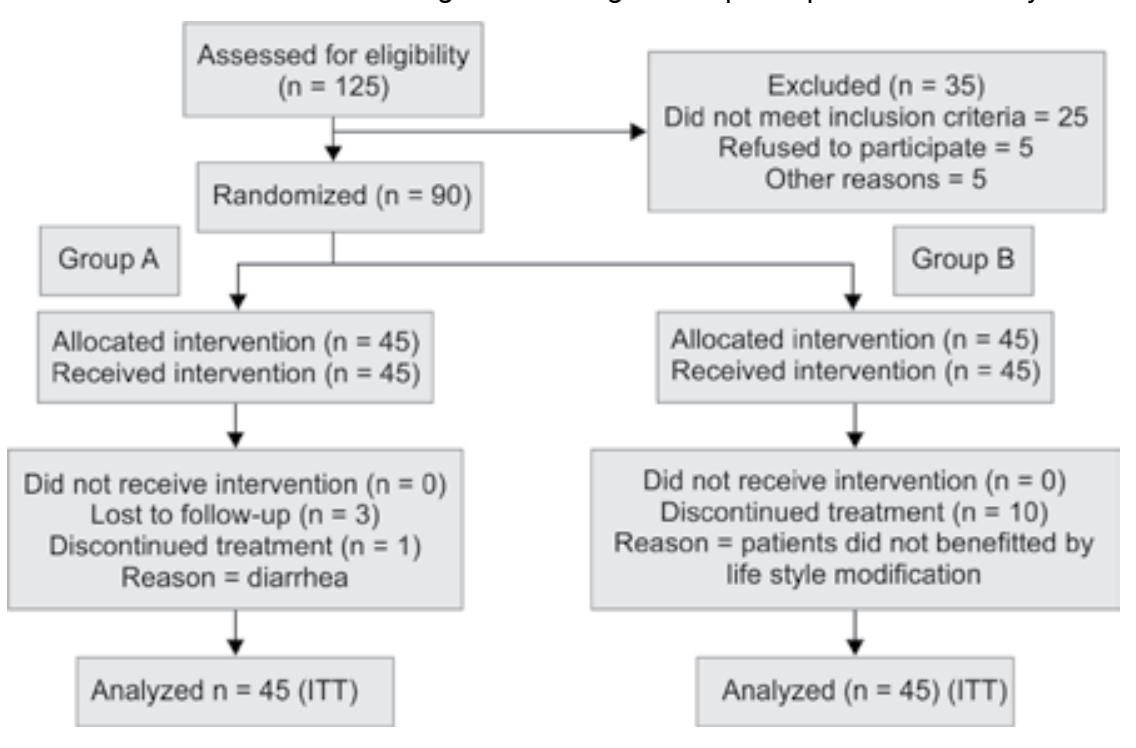

compared to group B. The most common presenting symptom was hoarseness of voice in both groups. At the end of 8 weeks RSI significantly decreased in both groups (Table 2). The percentage reduction at 8 weeks was higher in group A compared to group B $(\mathrm{p}<0.05)$ (Graph 1).

Individual symptoms like hoarseness of voice, excess throat mucus, clearing of throat, dry cough and foreign body sensation in the throat were reduced in both the groups (Table 3). The reduction in symptoms among the two groups was not statistically significant. However, greatest percentage reduction was seen in excess throat mucus and clearing of throat in group A compared to group B.

The RFS was $9.80 \pm 0.34$ in group A and $9.17 \pm 0.29$ in group $B$ at baseline. The RFS reduced in both groups of patients although, the reduction was not as high as

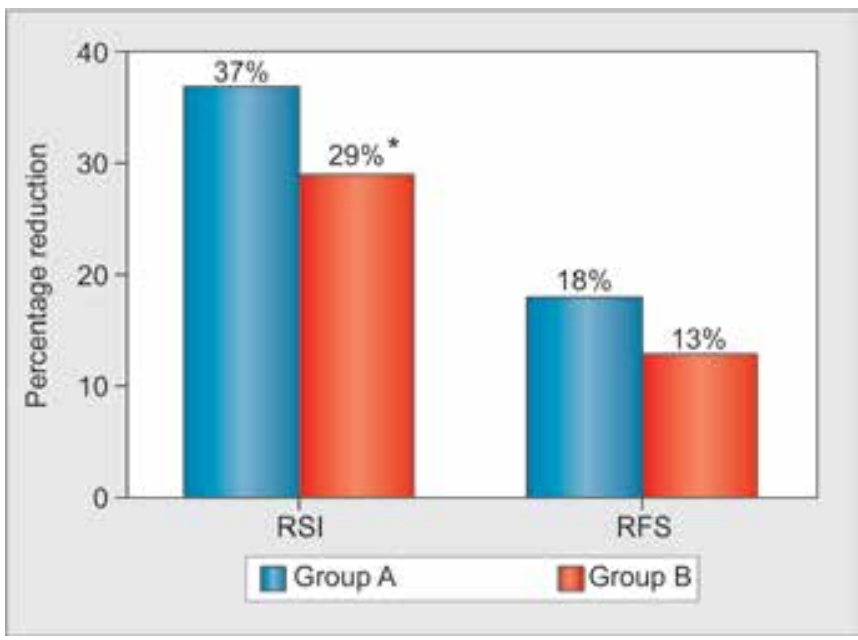

Graph 1: Percentage reduction in RSI and RFS in both groups at baseline and 8 weeks (group A: life style modification + PPIs)
RSI. After 8 weeks of treatment percentage reduction of RFS is shown in Graph 1. Intergroup analysis of RFS in terms of absolute values and percentage reduction was not statistically significant. In subgroup analysis, we evaluated the signs of LPRD like erythema, posterior laryngitis, vocal cord edema, laryngeal edema and granulations in individual patients (Table 3). The reduction in arytenoid erythema was seen more in group A as compared to group B. Although, the difference was not statistically significant, it was appreciable by comparing percentage reduction.

The mean change in the QoL score suggested an improvement in QoL in both groups. However, when the improvement in score attained by omeprazole and that attained by control group after 8 weeks was compared, no statistically significant difference was found (Graph 2).

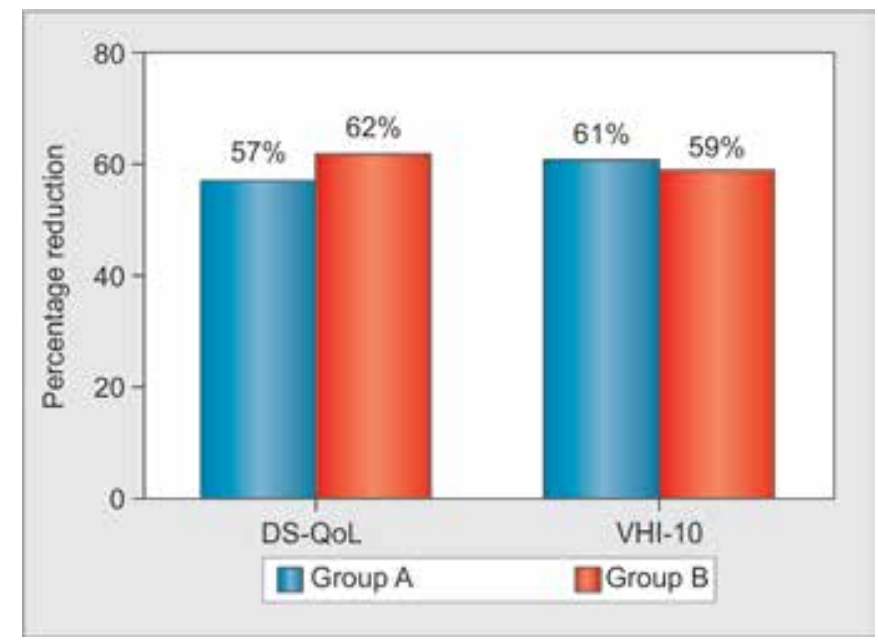

Graph 2: Percentage reduction in DS-QoL and $\mathrm{VHI}-10$ in both groups at baseline and 8 weeks (group B: life style modification alone)

${ }^{*} \mathrm{p}<0.05$ as compared to group $A$ 
Table 2: Clinical characteristics of patients in both the groups at 0 and 8 weeks

\begin{tabular}{llllll}
\hline & \multicolumn{2}{c}{ Group $A(n=45)$ omeprazole } & & \multicolumn{2}{c}{ Group $B(n=45)$ life style modification } \\
\cline { 2 - 3 } \cline { 5 - 6 } Characteristics & 0 week & 8 weeks & & O week & 8 weeks \\
\hline Reflux symptom index & $18.24 \pm 0.77$ & $11.49 \pm 0.65^{*}$ & & $17.56 \pm 0.68$ & $12.38 \pm 0.55^{*}$ \\
Reflux finding score & $9.80 \pm 0.34$ & $8.00 \pm 0.24^{*}$ & & $9.18 \pm 0.29$ & $7.91 \pm 0.20^{*}$ \\
Quality of life & $9.09 \pm 0.74$ & $5.15 \pm 0.61^{*}$ & & $8.90 \pm 0.65$ & $5.48 \pm 0.60^{*}$ \\
Voice handicap index & $11.22 \pm 0.36$ & $6.92 \pm 1.06^{*}$ & & $9.35 \pm 0.75$ & $5.48 \pm 0.57^{*}$ \\
\hline
\end{tabular}

All values are in mean \pm SE (standard error); ${ }^{*} p$-value $<0.05$ as compared to baseline; $p$-value $<0.05$ as compared to group $\mathrm{A}$

Table 3: Subgroup analysis of RSI and RFS

\begin{tabular}{|c|c|c|c|}
\hline & Week (s) & Group A & Group B \\
\hline \multicolumn{4}{|l|}{ RSI symptoms } \\
\hline \multirow[t]{2}{*}{ Hoarseness } & 0 & $3.17 \pm 0.18$ & $3.22 \pm 0.17$ \\
\hline & 8 & $1.88 \pm 0.13^{*}$ & $2.00 \pm 0.14^{*}$ \\
\hline \multirow[t]{2}{*}{ Excess throat mucus } & 0 & $2.77 \pm 0.22$ & $2.77 \pm 0.22$ \\
\hline & 8 & $1.28 \pm 0.15^{\star}$ & $1.77 \pm 0.14^{*}$ \\
\hline \multirow[t]{2}{*}{ Clearing of throat } & 0 & $2.91 \pm 0.22$ & $3.15 \pm 0.17$ \\
\hline & 8 & $1.40 \pm 0.16^{*}$ & $2.11 \pm 0.15^{*}$ \\
\hline \multirow[t]{2}{*}{ Something sticking } & 0 & $2.42 \pm 0.23$ & $2.17 \pm 0.20$ \\
\hline & 8 & $1.51 \pm 0.16^{*}$ & $1.35 \pm 0.4^{*}$ \\
\hline \multirow[t]{2}{*}{ Swallowing/breathing } & 0 & $2.28 \pm 0.19$ & $2.00 \pm 0.21$ \\
\hline & 8 & $1.24 \pm 0.13^{*}$ & $1.26 \pm 0.15^{\star}$ \\
\hline \multicolumn{4}{|l|}{ RFS symptoms } \\
\hline \multirow[t]{2}{*}{ Erythema } & 0 & $2.02 \pm 0.06$ & $1.82 \pm 0.10$ \\
\hline & 8 & $1.75 \pm 0.07^{*}$ & $1.48 \pm 0.10^{*}$ \\
\hline \multirow[t]{2}{*}{ Vocal fold edema } & 0 & $1.75 \pm 0.09$ & $1.88 \pm 0.1$ \\
\hline & 8 & $1.46 \pm 0.07^{*}$ & $1.46 \pm 0.09^{\star}$ \\
\hline \multirow[t]{2}{*}{ Laryngeal edema } & 0 & $1.66 \pm 0.11$ & $2.06 \pm 0.13$ \\
\hline & 8 & $1.15 \pm 0.10^{*}$ & $1.55 \pm 0.10^{*}$ \\
\hline \multirow[t]{2}{*}{ Posterior laryngitis } & 0 & $2.06 \pm 0.11$ & $1.80 \pm 0.15$ \\
\hline & 8 & $1.51 \pm 0.09^{*}$ & $1.31 \pm 0.1^{*}$ \\
\hline \multirow{2}{*}{$\begin{array}{l}\text { Granulation/thick } \\
\text { mucus }\end{array}$} & 0 & $0.82 \pm 0.14$ & $0.46 \pm 0.11$ \\
\hline & 8 & $0.83 \pm 0.11$ & $0.33 \pm 0.08^{*}$ \\
\hline
\end{tabular}

All values are in mean \pm SE (standard error); ${ }^{*}$ p-value $<0.05$ as compared to baseline; $p$-value $<0.05$ as compared to group $A$

Furthermore, voice handicap index-10 (VHI-10) was also significantly reduced in both groups without any significant difference between both groups.

There were no serious adverse events reported. Only three patients in group A had complaints of abdominal pain, constipation and diarrhea. One patient was withdrawn from group A (omeprazole) because of diarrhea. Patient recovered fully with treatment. Similarly, out of total of 45 patients in group B, 10 patients were not able to follow life style modification or did not show adequate response after 4 weeks. They were administered treatment in the form of other PPIs like rabeprazole 20 $\mathrm{mg}$ and pantoprazole $20 \mathrm{mg}$. To analyze these patients, the LOC forward method was used.

\section{DISCUSSION}

The baseline demographic characteristics, such as age and gender were comparable in both groups. Randomized studies in other countries included patients with a mean age that was higher than that of patients in our study. $8,10,17$ It may indicate that Indian patients are affected at a younger age than other populations. Moreover, LPRD was distributed almost equally among males and females which was comparable to earlier studies. ${ }^{7,8}$ Hoarseness of voice was the most common symptom observed in this study, which is also seen in large $(n=225)$ randomized trial of LPRD. ${ }^{18}$ This confirms that there is not much difference in presentation of patients between different populations. In this study, at baseline, only $37 \%$ of LPRD patients had acid reflux which is comparable to western literature (30-45\%).

We saw reduction of RSI and RFS over a period of time. However, like other studies, the mean score reduction was more in RSI compared to RFS. ${ }^{7}$ Inter-group comparison in reduction of both scores was statistically nonsignificant. Although, in general, omeprazole group patients fare better than life style modification group.

To see the difference between two treatment modalities, we applied percentage reduction in RSI and RFS. We observed that percentage reduction of RSI was significantly higher in omeprazole group compared to life style modification group $(\mathrm{p}<0.05)$. So, it implies that there was a trend toward improvement in omeprazole group compared to life style modification group. This result correlates with other studies done with other PPIs ${ }^{10,19}$ One randomized, double-blind, placebo-control trial of lansoprazole (30 mg twice daily) for 3 months found improvement in a significantly greater proportion in lansoprazole treated $(50 \%)$ subjects than the placebo group $(10 \%) .{ }^{10}$ Moreover, in subgroup analysis of different symptoms, improvement was seen equivalently in all the parameters in both groups. However, interestingly percentage reduction of excess throat mucus and clearing of throat were more in omeprazole group compared to other. This finding correlates with other studies in which above mentioned symptoms reduced considerably compared to others. ${ }^{7,9}$ 
Even after 2 months of treatment RFS decreased only by 1 point in both groups. None of the patients showed complete resolution of any sign. Absolute values of mean reduction were comparable between two groups. Although, when we analyzed percentage reduction in RFS score between two groups, omeprazole group was superior compared to other group. These findings suggest that, the life style modification does have impact on LPRD, but when we added it with PPIs, it has additive outcome. Percentage reduction of arytenoid erythema was higher in patients who were taking omeprazole and life style modification than life style modification alone. Improvement in other signs was equivocal and similar in both groups. Most of the randomized studies have shown that there was not much difference in improvement in laryngeal signs between the groups. The reason behind very minor improvement is due to the duration of studies as most of the randomized trials are of 2 to 3 months duration. ${ }^{10,20}$ We are not able to see large improvement in laryngeal signs, because it needs at least 6 months to improve. Studies have shown that symptoms improved before signs and because of the same reason American Association of Otolaryngology-head and neck surgery (AAO-HNS) has recommended 6 months of PPIs use in LPR patients. ${ }^{21}$

Interestingly, we observed sizable number of patients $(22 \%)$ had to cross over from groups B to A after 4 weeks due to lack of benefit from life style modification only. This finding suggests that life style modification only has inferior outcomes compared to when given with PPIs.

We used disease specific quality of life (DS-QoL) and VHI-10 to evaluate the impact of LPRD on QoL and voice. In this study, DS-QoL and VHI-10 improved in both groups after the study duration. However, no significant difference regarding the effects of the two different treatments instituted under the two groups were appreciated on the VHI scores and QoL. It suggests that life style modification also has a role in improvement of voice in the same way as observed in other studies. ${ }^{22,23}$ Omeprazole was well tolerated in our study. There were few cases of nausea, constipation and diarrhea. One patient of omeprazole group in our study was removed because of diarrhea. Earlier studies also mentioned the lack of any significant ADRs due to PPIs. ${ }^{24,25}$

In conclusion, the results indicate that omeprazole when given with life style modification will be more beneficial and accepted than life style modification alone in LPRD.

Ethical approval: All procedures performed in studies involving human participants were in accordance with the ethical standards of the institutional and/or national research committee and with the 1964 Helsinki declaration and its later amendments or comparable ethical standards.
Informed consent: Informed consent was obtained from all individual participants included in the study.

\section{ACKNOWLEDGMENT}

We would like to acknowledge the help of Mr Sanjeev, audiologist and speech therapist of department of otolaryngology for providing speech therapy to study patients.

\section{REFERENCES}

1. Ford CN. Evaluation and management of laryngopharyngeal reflux. J Am Med Assoc 2005;294(12):1534-1540.

2. Sen P, Georgalas C, Bhattacharyya AK. A systematic review of the role of proton pump inhibitors for symptoms of laryngopharyngeal reflux. Clin Otolaryngol 2006;31(1):20-24.

3. Belafsky PC, Postma GN, Koufman JA. Validity and reliability of the reflux symptom index. J Voice 2002;16(2):274-277.

4. Belafsky PC, Postma GN, Koufman JA. The validity and reliability of the reflux finding score. Laryngoscope 2001; 111(8):1313-1317.

5. Martinucci I, de Bortoli N, Savarino E, Nacci A, Romeo SO, Bellini $\mathrm{M}$, et al. Optimal treatment of laryngopharyngeal reflux disease. Ther Adv Chronic Dis 2013;4(6):287-301.

6. Klok RM, Postma MJ, van Hout BA, Brouwers JR. Metaanalysis: comparing the efficacy of proton pump inhibitors in short-term use. Aliment Pharmacol Ther 2003;17(10): 1237-1245.

7. Noordzij JP, Khidr A, Evans BA, Desper E, Mittal RK, Reibel JF, et al. Evaluation of omeprazole in the treatment of reflux laryngitis: a prospective, placebo-controlled, randomized and double-blind study. Laryngoscope 2001;111(12):2147-2151.

8. Vaezi MF, Richter JE, Stasney CR, Spiegel JR, Iannuzzi RA, Crawley JA, et al. Treatment of chronic posterior laryngitis with esomeprazole. Laryngoscope 2006;116(2):254-260.

9. Lam PK, Ng ML, Cheung TK, Wong BY, Tan VP, Fong DY, et al. Rabeprazole is effective in treating laryngopharyngeal reflux in a randomized placebo-controlled trial. Clin Gastroenterol Hepatol 2010;8(9):770-776.

10. El-Serag HB, Lee P, Buchner A, Inadomi JM, Gavin M, McCarthy DM. Lansoprazole treatment of patients with chronic idiopathic laryngitis: a placebo-controlled trial. Am J Gastroenterol 2001;96(4):979-983.

11. Wineland AM, Piccirillo JF. Proton pump inhibitors versus placebo in the treatment of LPR: a meta-analysis of nine RCTs. Otolaryngol Head Neck Surg 2013;149:P88-P88.

12. Gill GA, Johnston N, Buda A, Pignatelli M, Pearson J, Dettmar PW, Koufman J. Laryngeal epithelial defenses against laryngopharyngeal reflux: investigations of E-cadherin, carbonic anhydrase isoenzyme III and pepsin. Ann Otol Rhinol Laryngol 2005;114(12):913-921.

13. Bulmer DM, Ali MS, Brownlee IA, Dettmar PW, Pearson JP. Laryngeal mucosa: its susceptibility to damage by acid and pepsin. Laryngoscope 2010;120(4):777-782.

14. Ethical guidelines for biomedical research on human participants. New Delhi, India 2006. Available at: http://icmr. nic.in/ethical_guidelines.pdf. Accessed on 09/04/2015.

15. World medical association declaration of helsinki: ethical principles for medical research involving human subjects. J Am Med Assoc 2013;310(20):2191-2194. 
16. Rosen CA, Lee AS, Osborne J, Zullo T, Murry T. Development and validation of the voice handicap index-10. Laryngoscope 2004;114(9):1549-1556.

17. Deary IJ, Webb A, Mackenzie K, Wilson JA, Carding PN. Short, self-report voice symptom scales: psychometric characteristics of the voice handicap index-10 and the vocal performance questionnaire. Otolaryngol Head Neck Surg 131(3):232-235.

18. Koufman JA. The otolaryngologic manifestations of gastroesophageal reflux disease (GERD): a clinical investigation of 225 patients using ambulatory 24-hour $\mathrm{pH}$ monitoring and an experimental investigation of the role of acid and pepsin in the development of laryngeal injury. Laryngoscope 1991;101 101(4 Pt 2 Suppl 53):1-78.

19. Langevin S, Ngo H. GERD-induced ENT symptoms: a prospective placebo controlled study with omeprazole $40 \mathrm{mg}$ a day. Gastroenterol 120(5):A16.

20. Eherer AJ, Habermann W, Hammer HF, Kiesler K, Friedrich G, Krejs GJ. Effect of pantoprazole on the course of reflux-associated laryngitis: a placebo-controlled doubleblind crossover study. Scand J Gastroenterol 2003;38(5): 462-467.

21. Koufman JA, Aviv JE, Casiano RR, Shaw GY. Laryngopharyngeal reflux: position statement of the committee on speech, voice, and swallowing disorders of the American Academy of Otolaryngology-head and neck surgery. Otolaryngol Head Neck Surg 2002;127(1):32-35.

22. StewardDL, WilsonKM,KellyDH,PatilMS,SchwartzbauerHR, Long JD, et al. Proton pump inhibitor therapy for chronic laryngo-pharyngitis: a randomized placebo-control trial. Otolaryngol Head Neck Surg 2004;131(4):342-350.

23. Koufman J, Sataloff RT, Toohill R. Laryngopharyngeal reflux: consensus conference report. J Voice 1996;10(3):215-216.

24. Reilly JP. Safety profile of the proton-pump inhibitors. Am J Health Syst Pharm 1996;56(23 suppl 4):S11-17.

25. Gerson LB, Triadafilopoulos G. Proton pump inhibitors and their drug interactions: an evidence-based approach. Eur J Gastroenterol Hepatol 2001;13(5):611-616. 